\title{
Wealth and health
}

\author{
Will the global credit crunch cause a health crunch in the most affected countries?
}

$\mathrm{H}$ uman lifespan in the developed world has been increasing continually for over a century, driven by steady medical advances coupled with, among other factors, improvements in diet and lifestyle on the back of rising prosperity. But this last part has a corollary: there must be some downward pressure on lifespan during times of declining prosperity. Indeed, some experts worry that mortality might increase, at least temporarily, in some countries where economies have collapsed as a result of the credit crunch.

Such fears seem well grounded given recent historical experiences such as the marked fall in life expectancy in some of the former Soviet States immediately after the collapse of communism. One well-studied case has been Kazakhstan where a rapid rise in mortality during the sharp economic deterioration in the early 1990s was followed by a tentative recovery in health and life expectancy as conditions improved after 1995 (Becker \& Urzhumova, 2005).

\section{...some experts worry that mortality might increase, at least temporarily, in some countries where economies have collapsed as a result of the credit crunch}

Most European countries are now better placed to weather the recession than the ex-Soviet states were in the 1990s, given the financial support from the EU to maintain basic levels of subsistence. In any case, economic measures alone are crude predictors of health and longevity, for they are mediated through lifestyle factors such as trends in diet and the incidence of smoking. Indeed, although the richer countries of Western Europe might be insulated to some extent against the direct health impact of the credit crunch, they have not been immune to other detrimental trends, notably rising levels of obesity and, in some cases, increased smoking among young women.

T he potential for lifestyle factors to derail long-term increases in life expectancy has been evident for some time. During the 1990s, mortality from lung cancer caused by smoking increased among women in France and Germany by $51 \%$ and $29 \%$, respectively (WHO, 2000). There has also been a narrowing in the difference between female and male life expectancy across all developed countries owing to a reduced difference in the numbers of male and female smokers, according to Sam Preston, author of several studies on the impact of smoking on longevity at the University of Pennsylvania's Population Centre (Philadelphia, PA, USA).

There is similarly firm evidence of the link between heavy drinking and mortality, largely from Russia both before and after the fall of communism. One recent study suggests that half of all deaths among working-age men in Russian cities are still caused by heavy drinking (Leon et al, 2007). Another study showed that hazardous drinking had an almost equally large impact on life expectancy in Eastern Europe (McKee \& Shkolnikov, 2001).

Meanwhile, heavy drinking has also been on the rise in parts of Western Europe, notably in the UK. One study revealed a steep increase in liver cirrhosis mortality rates in Scotland during the 1990s (Leon \& McCambridge, 2006), although more recent data suggest that the incidence of binge drinking among women, although not negligible, had been exaggerated in the media (Emslie et al, 2009).

As Adam Drewnowski at the University of Washington (Seattle, WA, USA) showed, there is also a link between metabolic conditions-notably obesity-and income (Monsivais \& Drewnowski, 2009). In essence, a healthy diet of fruits and vegetables usually costs more than fast food; to save money, people eat cheaper food that is usually higher in carbohydrates and thus increases their risk of obesity.

More generally, the twin factors of an unhealthy diet and insufficient exercise can exert a negative influence on health among all socio-economic groups, and increase levels of obesity. There is strong evidence that even being moderately overweight increases the risk of developing a cluster of conditions known as metabolic syndrome, which includes insulin resistance, hypertension and dyslipidaemia-disrupted levels of lipids including fats, fatty acids and cholesterol. Metabolic syndrome leads readily to type II diabetes and vascular diseases, with an increased risk of fatal heart attacks, strokes and some cancers.

\section{...to save money, people eat cheaper food that is usually higher in carbohydrates and thus increases their risk of obesity}

Furthermore, there is growing evidence that metabolic conditions, especially obesity and type II diabetes, can be passed on to children by epigenetic inheritance, suggesting that their incidence could continue to rise even after the root causes of poor diet and lack of exercise have been addressed. Several studies have reported that mothers who are diabetic or overweight during pregnancy - often as a result of high-calorie diets-are more likely to have obese children (Mayer-Davis et al, 2006). There is also accumulating evidence in animal models that metabolic conditions, including obesity and type II diabetes, resulting from 


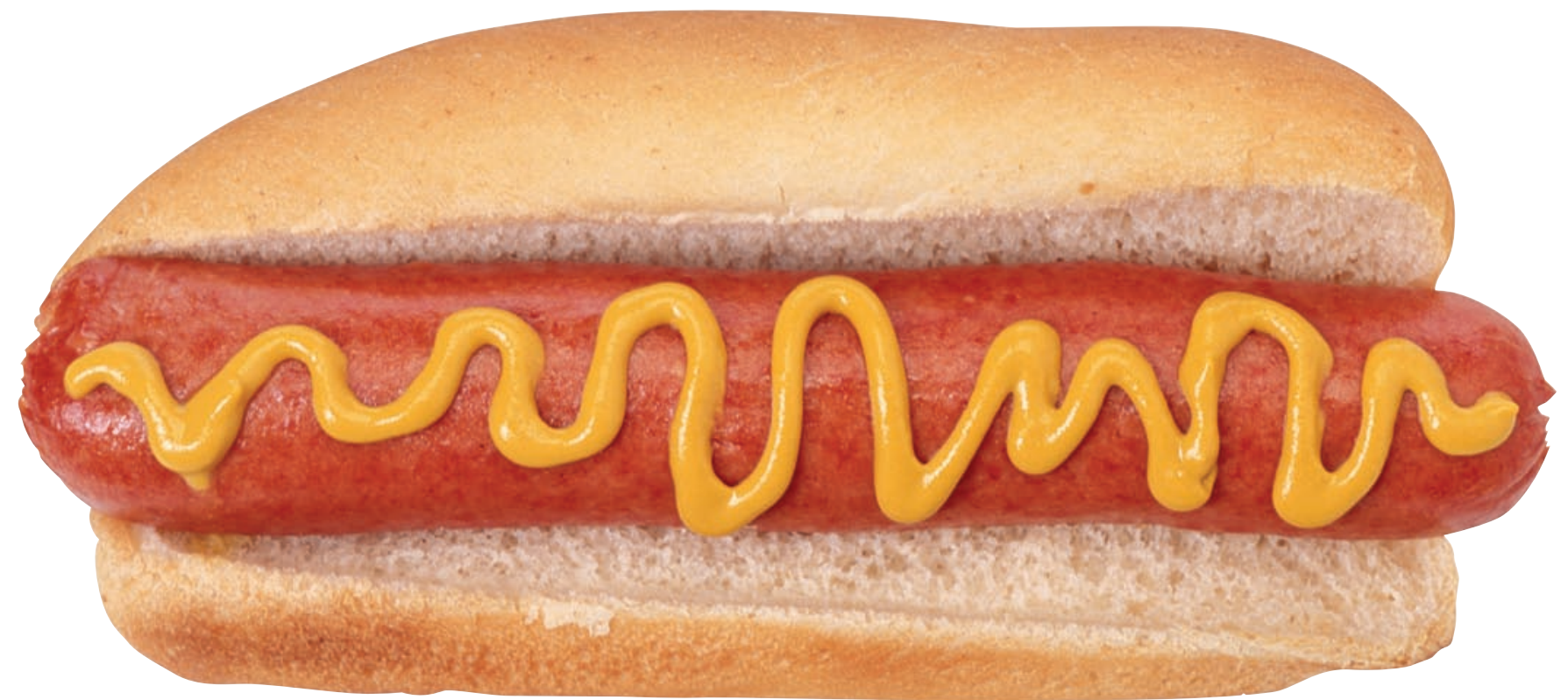

epigenetic reprogramming in response to environmental factors, can be passed on to several more generations at least.

$\mathrm{H}$ owever, the causal link between obesity and life expectancy remains uncertain and controversial; although few deny that obesity is on the rise, not all researchers agree that this translates automatically into a significant increase in mortality among any particular age group. "I do not expect a decline in longevity," said Preston. "In fact, the large increase in obesity, coinciding with and related to a reduction in smoking, has not had any apparent effect on the steady improvement in longevity that has occurred over the same period." He is hinting that a significant proportion of ex-smokers turn to eating instead, which has been supported by several studies showing both that ex-smokers, and animals forced to imbibe smoke, change their diet to one higher in carbohydrates upon the sudden withdrawal of nicotine. Preston's point is that, on average, people who are substituting smoking for eating are replacing one evil with a lesser one in health terms, which leads to a net increase in life expectancy. Obesity might still be bad for you, but not as bad as smoking.

But the picture is rather more complex, according to Neil Mehta, a graduate student who is working with Preston on studies of 'longitudinal' data about obesity and mortality, which track people over time. His data indicate that, unlike smoking, the impact of obesity is not entirely negative. "We must have a more nuanced appreciation of the relationship between obesity and mortality-it is very complex and depends on a number of factors, most notably age. For middle and older-aged adults there appears to be a slight protective effect of being overweight [...] but not obese," said Mehta. "So-called 'mild' obesity [..] shows little increased risk of death compared to 'normal' [...] among adults—some studies actually show no increased risk of death for 'mild' obesity." Mehta argues therefore that as most adults lie in the mild category, the impact on average lifespan is negligible. Although moderate or severe obesity are associated with increased mortality, only a small percentage of the population are in that category.

\section{...the twin factors of an unhealthy diet and insufficient exercise can exert a negative influence on health among all socio-economic groups, and increase levels of obesity}

Mehta's analysis implies that somehow the final link between moderate obesity and premature death has been broken. "One possible explanation is that we are doing something right to prevent death, but that we have been less successful at preventing obesity-related illnesses and disability," he said. "In my current and ongoing work, I show a very clear decline in the effect of obesity on mortality in the United States since the 1970s. The declining effect is really due to a reduction in the obesity-related risk of dying from heart disease. So, one possible explanation is that recent improvements in heart disease treatments have done a good job at keeping obese people with cardiovascular conditions alive."

7 he question is then how to improve public health, not only during but also beyond the financial and economic crisis. For smoking and binge drinking, the remedy is obvious. But obesity and related diseases have been harder to tackle even if obese people do not enjoy the same quality of life as those of normal weight, and are more prone to various disabilities in addition to reduced well-being and self esteem. There is therefore good reason for tackling obesity, quite apart from any impact on mortality and lifespan. Yet, it so happens that most therapies or regimes that counter obesity in animal models also increase life expectancy, often markedly.

Such therapies come in two main categories: either by reducing energy supply through cutting calorie intake or by increasing energy consumption, which can be accomplished by adding an appropriate component to the diet or through exercise. 
The energy supply side has been studied in greatest depth, with strong evidence that calorific reduction achieves a universal increase in lifespan across a wide range of organisms from yeast to rodents. More recently, there has also been accumulating evidence that diets comprising significantly reduced levels of the amino acid methionine achieve an almost equally marked increase in lifespan in mice and rats, while reducing weight. In fact, if the effects were additive-in other words, a diet low both in calories and methionine-it could have a marked effect on human life expectancy. Furthermore, as exercise regimes seem to achieve similar effects, it can be asked whether they have a further additive component on lifespan.

There is some disagreement, however, when it comes to comparing and contrasting calorie and methionine restriction, and whether they are additive or merely alternative strategies operating through the same pathways. Thomas Gettys, Professor of Nutrient Sensing and Adipocyte Signaling at Louisiana State University (Baton Rouge, LA, USA) argued that the effects are not cumulative, with methionine and calorie restriction being alternative routes to the same outcome. Although the actual pathways are different, both work by reducing the amount of adipose tissue, Gettys suggested, but the exact mechanisms have yet to be elucidated. "Reduction in adiposity is something calorie restriction shares with methionine restriction," said Gettys, "along with a whole series of other common effects. For example they both cause a significant increase in adiponectin (a protein hormone secreted by adipose cells), which is involved in insulin sensitivity but also in fuel oxidation." The difference is that while calorie restriction reduces energy supply, methionine reduction seems to boost energy expenditure. "So they both affect homeostasis in different ways," said Gettys.

M ore work needs to be carried out, however, with the ultimate prize of developing a drug that achieves the same impact without the need to observe a stringent diet-as Gettys pointed out, for humans neither methionine nor calorie restriction are easy to sustain over a long period. Some candidate compounds are already emerging that might be able to reverse obesity and boost lifespan in the same manner as calorie- or methionine-restricted diets. A study by David Sinclair and colleagues at Harvard Medical School (Cambridge, MA,
USA) found that the detrimental effect of a diet high in calories and fat could be completely eliminated by administering a drug containing resveratrol (Milne et al, 2007), one of a group of antibiotics called phytoalexins produced by some plants when under attack from bacteria or fungi.

\section{...although few deny that obesity is on the rise, not all researchers agree that this translates automatically into a significant increase in mortality among any particular age group}

"Our work in mice shows it is possible to slow down many diseases of aging with a single drug," said Sinclair. "We hope that is true in humans too." But Sinclair cautioned that until the root causes and effects are fully unravelled, it will not be possible to predict the actual impact of such therapies on human lifespan or prescribe them with total confidence of safety. But hopes are rising that drug interventions might be able to reduce the incidence of metabolic conditions, particularly obesity. This, in turn, could help to reduce the health and lifespan inequality between socio-economic groups and countries, and might also help to avoid future health catastrophes such as the one that struck the ex-Soviet states in the early 1990 s.

A nother big question is whether such therapies can boost human lifespan at the top end of the scale and reduce mortality among the very elderly. This is significant because although average human lifespan has increased enormously in developed countries, mortality rates among the very elderly have remained stubbornly constant. There has been little confidence that the marked increases in maximum lifespan achieved among laboratory animals through caloric or methionine restriction can be replicated in humans.

However, the data suggests such pessimism might be unfounded, according to Roland Rau, from the Laboratory of Survival and Longevity at the Max Planck Institute for Demographic Research in Rostock, Germany. "It has long been considered that nothing can be done about mortality at advanced ages," he said. "We have seen, though, that mortality has been dropping among the very elderly since the 1950s and at an accelerating pace in some countries since the 1970s. For example, the probability that an 80-year-old woman will celebrate her $90^{\text {th }}$ birthday was on average about $15 \%$ in the 1950 s (looking at most Western European countries, Canada, the US, Australia, New Zealand and Japan). In 2002, the corresponding number was approximately $37 \%$. For Japanese women it was even more than 50\%" (Rau et al, 2008). At the same time, records for extreme longevity beyond 110 continue to be broken so, if there is a maximum, it has yet to be determined precisely. "Most think such a maximum exists, but evidence from the steady improvement in life expectancy achieved by the best performers shows that it is still at a distance," said Rau.

In any case, although the pursuit of longer lives of course holds much fascination both for scientists and the general public, the real benefits of new therapies that address obesity or metabolic disorders are to improve the quality of life among people of all ages and to reduce the incidence of debilitating disabilities.

\section{REFERENCES}

Becker CM, Urzhumova DS (2005) Mortality recovery and stabilization in Kazakhstan. Econ Hum Biol 3: 97-122

Emslie C, Lewars H, Batty GD, Hunt K (2009) Are there gender differences in levels of heavy, binge and problem drinking? Evidence from three generations in the west of Scotland. Public Health 123: 12-14

Leon DA, McCambridge J (2006) Liver cirrhosis mortality rates in Britain from 1950 to 2002: an analysis of routine data. Lancet 367: 52-56

Leon DA, Saburova L, Tomkins S, Andreev EM, Kiryanov N, McKee M, Shkolnikov VM (2007) Hazardous alcohol drinking and premature mortality in Russia: a population based casecontrol study. Lancet 369: 9578

Mayer-Davis EJ, Rifas-Shiman SL, Zhou L, Hu FB Colditz GA, Gillman MW (2006) Breast feeding and risk for childhood obesity: does maternal diabetes or obesity status matter? Diabetes Care 29: 2231-2237

McKee M, Shkolnikov V (2001) Understanding the toll of premature death among men in Eastern Europe. BM/ 323: 1051-1054

Milne JC et al (2007) Small molecule activators of SIRT1 as therapeutics for the treatment of type 2 diabetes. Nature 450: 712-716

Monsivais P, Drewnowski A (2009) Lowerenergy-density diets are associated with higher monetary costs per kilocalorie and are consumed by women of higher socioeconomic status. J Am Diet Assoc 109: 814-822

Rau R, Soroko E, Jasilionis D, Vaupel JW (2008) Continued reductions in mortality at advanced ages. Popul Dev Rev 34: 747-768

WHO (2000) The World Health Report 2000. Geneva, Switzerland: World Health Organization

\section{Philip Hunter}

doi:10.1038/embor.2009.171 